\title{
Programa Razonado de Introducción a la Historia Universal
}

Por ser un curso de "cultura general", no debe limitarse:

a), ni al estudio del carácter, método y sistemas de interpretación histórica, pues ello implicaría su dedicación a los futuros especialistas;

b), ni a una visión panorámica del desenvolvimiento histórico de los principales pueblos, porque tal es el objeto de los cursos escolares;

$\mathrm{y}$, en consecuencia, será dedicado a la exposición y crítica de las formas de vida que señalan y caracterizan las principales etapas del progreso cultural y social de la humanidad.

\section{Biblio preliminares}

Según una definición clásira, que la considera como género literario: la HISTORIA es una narración verídica y ordenada de los hechos pasados, que han influído en el destino de la humanidad.

Pero, si prescindimos de su carácter narrativo, que sólo afecta a la forma de exposición, y no al contenido de la historia, hallamos en dicha definición los siguientes requisitos exigidos al relato histórico:

$a$, veracidad y orden;

b), restauración de los hechos pasados; y

c), crítica y selección de éstos, a fin de tratar únicamente los que han influído en el destino de la humanidad y evitar cuantos carezcan de significado trascendente.

No aparece, ni indirectamente, la libertad creadora que anima el trabajo del artista literario. $Y$, por el contrario, todos los elementos de la definición suponen la investigación y el método como antecedentes de 
la construcción histórica. Luego la HISTORIA no puede ser asimilada a la condición de género literario. ES CIENCIA.

Ahora bien, en armonía con los principios de la Lógica -ciencia filosófica que norma el conocimiento- puede establecerse que:

a), "la ciencia sólo se refiere a lo general" (según Áristóteles), y la Historia efectúa estudios particulares (sobre el pasado de un pueblo determinado, por ejemplo) o especiales (desenvolvimiento de una institución o una categoría ideológica), para edificar sobre ellos la máxima construcción histórica, la historia de la civilización;

b), la ciencia se esfuerza por esclarecer la verdad de cada fenómeno (sea éste físico, biológico o social), y la Historia investiga para restaurar la verdad en cuanto atañe a los hechos que la humanidad vivió en el pasado;

c), la ciencia supone el "saber por las causas" (según Bacon), y la Historia tiende a determinar por qué sucedieron los hechos sometidos a su estudio; $y$, finalmente,

d), la ciencia persigue su objeto a través de una serie de procedimientos que constituyen el método, el cual se halla representado en la Historia por el estudio de las fuentes materiales o documentales, la crítica y ordenación de sus informaciones y la construcción histórica propiamente dicha.

Luego la Historia satisface las exigencias hechas a toda ciencia y, con mayor justeza que en la definición clásican se puede caracterizar su contenido en los términos siguientes: La Historia es una ciencia que tiene por objeto restaurar la verdad de los hechos pasados, en cuanto ejercieron alguna influencia sobre la vida y el destino de la humanidad.

La Historia en la clasificación de las ciencias.-En armonía con sus tendencias evolucionistas, Augusto Comte clasifica las ciencias atendiendo al orden histórico de su aparición y a la jerarquía determinada por la complejidad progresiva de su contenido. Distingue las siguientes ciencias fundamentales:
a) de los cuerpos inorgánicos $\left\{\begin{array}{l}\text { Matemáticas } \\ \text { Astronomía } \\ \text { Física } \\ \text { Química }\end{array}\right.$
b), de los cuerpos orgánicos
$\{$ Biología (o Fisiología)
\{ Física Social (Sociología e Historia) 
Para Herbert Spencer, que intentó enmendar la clasificación de Comte, las ciencias son: abstractas, abstracto-concretas y concretas. Al agrupar estas ulltimas obedece, como su antecesor, a un criterio evolutivo, jerárquico y de complejidad creciente, y distingue: Astronomía, Geología, Biología, Psicología y Sociología (e Historia).

Aparte la comprensión de los caracteres propios de la Historia, el lugar que se le señala en las clasificaciones de Comte y Spencer indica: 19, la relativa modernidad de la Historia; $2^{\circ}$, la complejidad de s11 contenido, ya que los resultados de la investigación histórica dependen en cierta manera de los datos proporcionados por las ciencias que la preceden en dichas clasificaciones; y $3 \%$, las estrechas relaciones existentes entre la Historia y la Sociología.

Historia y Sociología.-Ante los grávidos problemas sociales del siglo XIX, y la preeminencia conservada por las interpretaciones individualistas y la clásica narración de los hechos pasados, se forjó una artificiosa distinción entre Sociología e Historia, según la cual:

al campo de la Historia pertenecen los hechos sociales de naturaleza específica, concretos y estables; y

al campo de la Sociología pertenecen los hechos sociales de naturaleza genérica, abstractos e inestables.

De tal distinción se infería que la Historia pone al alcance de la Sociología los resultados de sus investigăciones, y ésta induce las leyes de los fenómenos sociales.

En verdad, la Historia proporciona a la Sociología sus informaciones sobre la formación y el desenvolvimiento de las sociedades $y$, en virtud del llamado método histórico, da origen a la Sociología genética; y, a su vez, la Sociología proporciona a la Historia el método sociológico, que considera el estudio sobre el carácter de las sociedades, las fuerzas que en ellas actúan y su equilibrio, etc.

Filosofía de la Historia e Historia.--La Filosofía de la Historia se esfuerza por identificar las "esencias" cuya permanencia aparece a través de los cambios sucesivos, $y$, en función de ellas, intenta fijar las leyes y el sentido del desenvolvimiento histórico. Es, pues, una interpretación de la Historia, un "modo de ver" el pasado. Y como el filósofo de la Historia se halla sometido a la influencia que sobre su ánimo detectan las condiciones sociales y culturales de su época, no existe sólo un "modo de ver" la Historia: existen puntos de vista subjetivos, o sistemas de interpretación, que al criterio del historiador se presentan como materia- 
les objetivos merced a los cuales conoce los ideales predominantes en una época dada.

Sistemas de interpretación histórica.-En forma sistemática, o contenidos en sus concepciones acerca del mundo y de la vida, filósofos e historiadores de todos los tiempos han expuesto sus "modos de ver" la Historia. Los principales son:

a), En la Grecia antigua, al lado de la filosofía dialéctica y bajo el influjo de una conciencia ahistórica, se concibe la sucesión y la repetición cíclica de los hechos históricos;

b), frente a la crisis del mundo antiguo y en armonía con sus ideales teológicos, San Agustín interpreta el destino histórico de la humanidad como el cumplimiento inexorable de los designios providenciales. Iniciado con el pecado original y la fundación de la ciudad terrena, se desenvuelve a través de una lucha incesante entre la libertad que lleva al pecado y la gracia divina que aparta de él, y conduce al hombre hacia La Ciudad de Dios, cuyo terrenal anticipo se halla en la iglesia. Fundamenta, así, la necesidad de que el poder político sirva a los fines de la iglesia, lo cual inspirć la política temporal de los papas durante las edades media y moderna, y se halla aplicado en el imperio carolingio y el Santo Imperio Romano-Germánico.

c), en su Discurso sobre la Historia Universal (1681), Bossuet -que vivió durante el auge de la monarquía absoluta y fué el sostenedor del derecho divino de los reyes-intentó explicar la Historia como una realización de la voluntad de Dios, e introdujo en la construcción histórica un principio de crítica, al seleccionar, entre los hechos pasados, aquellos que contribuyeran a comprobar su teoría.

d), providencialista es, también, la interpretación de Vico, quien fija el primer sistema de Filosofía de la Historia propiamente dicho, en sus Principios de una Ciencia Nueva en torno a la naturaleza común de las naciones (1730). En todos los pueblos distingue tres edades: infancia o salvajismo, juventud $\circ$ edad heroica, y madurez. En ésta realiza la humanidad sus anhelos, pero sobrevienen la corrupción y la disolución, y vuelve entonces a la primera edad, para seguir otra vez el mismo curso. Toda la historia la concibe, en consecuencia, como una ininterrumpida serie de corsi y recorsi. Y en la exposición de este esquema intu- 
ye, por primera vez, el vasto campo de la prehistoria, el contenido simbólico de la mitología, y la contribución que el conocimiento del pasado obtiene de las ciencias auxiliares (Lingüística, Geografía, Heráldica, Diplomática, Numismática).

e), tanto en la interpretación como en la elaboración de la Historia, queda abandonada la tendencia teológica y providencialista gracias al influjo que la razón alcanzó durante el período de la ilustración. La Historia se desvincula de la religión e inicia su estructuración como ciencia. En su Ensayo sobre las costumbres $y$ el espíritu de las naciones (1745-1750) Voltaire toma como objeto de la Historia los progresos del espíritu humano, a los que atribuye más hondo contenido y mayor eficacia ilustrativa que a los hechos políticos, pero no concibe que la razón haya sufrido cambios en su naturaleza. Inspiración semejante sigue Condorcet en su Bosquejo de un cuadro histórico de los progresos del espíritu humano (1795), al fi)ar las principales etapas de la evolución histórica en relación con el grado de adelanto de las ciencias y las artes, y al establecer que una mayor difusión de ellas haría más amplios los progresos del futuro próximo.

f), en todo su sistema filosćfico, pero principalmente en la Filosofía de la Historia y la Filosolía del Espíritu (1807), Hegel traza una interpretación dialéctica, cuyo acentuado carácter idealista la vincula en cierta manera a los sistemas providencialistas. Desde su principio, halla la causa de todos los eventos en la razón absoluta (o razón universal), cuya existencia concibe como anterior al hombre e independiente de él; por tendencia propia, dicha razón absoluta aspira a realizarse en la naturaleza y así origina su proceso de evolución, que crea la historia y como la razón absoluta ha de proponerse una finalidad, atribuye tal carácter al establecimiento del estado, con el cual se inicia la historia, y la realización de aquel aspecto particular de la razón absoluta que mayor armonía guarde con el espíritu particular del pueblo. Dialécticamente, explica el tránsito de una etapa a otra como un cambio de cantidad en calidad, y viceversa; o como un resultado de la oposición de contrarios que se da dentro de cada cosa o fenómeno. La interpretación hegeliana elimina, por lo tanto, el azar; fundamenta la causalidad del acontecer histórico, y fija sus leyes. 
g), aunque derivado de la dialéctica hegeliana, el materialismo histórico introduce una completa renovación en los fundamentos de la interpretación histórica. Sus fundadores fueron Carlos Marx y Federico Engels; pero la primera exposición de sus principios fué ofrecida por aquél en la "Contribución a la crítica de la Economía Política" (1859). "No es la conciencia del hombre lo que determina su existencia, sino su existencia social lo que determina su conciencia". Las reiaciones sociales de producción forman la estructura económica de la sociedad; y sobre ésta se levanta la superestructura jurídica y política y las superestructuras ideológicas. En la estructura económica se produce una evolución constante, de carácter cuantitativo, que da origen a un cambio cualitativo, el cual coincide, históricamente, con los "saltos" revolucionarios. Pero el desarrollo de las superestructuras ideológicas (religión, ciencia, cultura) puede anticiparse a la efectividad de aquellas cambios, y aun prepararlos: entre la estructura y las superestructuras existe, pues, un constante flujo y reflujo. Es claro, por esto, que entre todos los fenómenos sociales existe estrecha relación; constituyen una unidad, en la cual luchan incesantemente los contrarios, manteniendo un equilibrio que sólo se rompe con el "salto" o la negación de la negación.

Otros sistemas, surgidos durante el siglo XIX, carecen de la grandeza y la visión totalizadora que los anteriores intentaron alcanzar, y su valor se limita a la preferentel rehabilitación deeun elemento histórico. Cabe mencionar los que se basan en la influencia de la economía, los factores geográficos y la raza.

Con el materialismo histórico ha sido confundida, a veces, la interpretación económica de la Historia, que explica el desenvolvimiento de la humanidad mediante la fuerza determinante de los factores económicos. Pero, en verdad, la interpretación económica enfoca sólo parciai y mecánicamente la vida histórica de los pueblos, y su riguroso determinismo la aleja de la dialéctica materialista.

La interpretación geográfica de la Historia se sustenta, principalmente, en la importancia que los factores geográficos tuvieron en la vida de los pueblos antiguos $y$, desde luego, en el progreso alcanzado por el conocimiento de la naturaleza. Sus representantes son Buckle y Ratzel. 
La interpretación étnica de la Historia concibe el auge y la decadencia de los pueblos como resultado de las aptitudes raciales. $\mathrm{Su}$ inspiración aparece netarnente en el libro de Gobineau sobre La desigualdad de lus razas humanas; y, en el fondo, tiende a proporcionar argurnentos justificatorios a la dominación imperialista sobre los pueblos de Atrica y Asia, a los cuales se afectaba proteger.

Durante el primer tercio dei presente siglo alcanzó extraordinario auge La Decadencia de Osciciente, libro de Oswald Spengler en el cual aparece una visión del púsado cue, en rigor, debe ser estimada como una sociología histórica; juzga lús culturas corno organismos que cumplen un proceso vital y, a la luz de la experiencia proporcionada por la Historia, prevé una inminente periciitación de nuestra cultura occidental. $Y$ en años recientes Benedetto Croce concibió la Historia como una hazaña de la lucha por la libertad, reaccionando así a la opresión del fascismo y dando actualidad a la visión kantiana.

Método de la Hisloria.-En sús diversas fases, la investigación que el historiador efectúa, tiene por objeto establecer la verdad de los hechos pasados, en conformiciad con los datos de las fuentes históricas (monumentos y restos materiales, testirnonios y documentos). Comprende:

19), Eurística, o búsqueda de las fuentes, fuere cual fuere su índole;

2). Estudio de losicaracteres externos de dichas fuentes, a fin de establecer su autenticidad;

3\%). Hermenéutica, o análisis del espíritu y la letra de las informaciones obtenidas en las fuentes, con el objeto de evitar cuarto hubiere de subjetivo o personal en ellas y restituir la verdad de los hechos; y

4\%), la construcción histórica.

En el curso de tales operaciones metódicas, el origen y la índole de las fuentes puede requerir el auxilio de conocimientos pertenecientes a otras ciencias. El historiadcr tiene en éstas a las "ciencias auxiliares de la Historia".

Ciencias Auxiliares de la Historia.-Propiamente, no existen ciencias cuyos conocimientos sean auxiliares a toda investigación histórica; en verdad, cada historiador tendrá como auxiliares solamente aquellas ciencias que pueden esclarecer los problemas de su especialidad. Así, por ejemplo, la identificación de la fecha y la procedencia de un docu- 
mento puede basarse en el análisis químico de la tinta y de la composición del papel y, sin embargo, la Qumica no está incluída entre las ciencias usuaimente consideradas como auxiliares de la Historia.

Las principales son:

Geografía y Cronolouía --Calificadas en olro iiempo como los "ojos de la Historia" - cuyos particulares estucios permiten establecer con exactitud el lugar y la época en que sucedieron los hechos históricos, y satislacen asi los requisitos básicos de la Historia narrativa;

Antropología, que inicialmente se aplicó a estudiar al hombre corno especie, intenta extenderse hoy hacia los caracteres genericos de su tarea y su destino;

Étnografía, que abarca la descripción y clasiticación general de las razas humanas, su distribucion geogratica, y las manitestaciones de sus actividades sociaies y culturales;

Etnología, que estuaia cada raza en particular, atiende a sus caracteres fisicos, sus semejanzas y diferencias con otras razas, su tormación historica, los caracteres especiticos de sus creencias, usos e industrias, y las relaciones e interinfluencias que los anteriores aspectos acusan;

Arqueología, que estudia las construcciones, los monumentos y, en general, los restos materiales de la industria humana;

Paleontología, dedicada al estudio de los fósiles animales y vegetales, contribuye a precisar la antigüedad de las muestras de la industria humana halladas al lado de dichos fósiles;

Epigrafía, que estudia los signos recordatorios o las inscripciones de los monumentos u objetos de uso;

Paleografía, que tiene por objeto el estudio de las escrituras antiguas y la descifración de sus caracteres;

Lingüística, o estudio de las lenguas, a cuya luz puede establecerse las áreas de expansión e influencia de los jueblos, así como la intensidad de las relaciones entre unos y otros;

Heráldica y Genealogía, que estudian los blasones y los linajes, respectivamente;

Numismática, a la cual corresponde el estudio de los problemas concernientes a las monedas y las medallas; 
Diplomática, que estudia los diplomas y documentos oficiales; y Folklore (del inglés folk = pueblo, lore = erudición), moderna disciplina que recoge, sistematiza y estudia las diversas formas de la cultura popular (creencias, usos, cantares, etc.), a fin de esclarecer su origen y significado.

Divisiones de la Historia.---Desde antiguo se hizo necesario parcelar el vasto dominio de la Historia, tanto para acentuar la especialización . de las investigaciones en lo referente a tal o cual de sus fases, como para orientar y ordenar la iniciación de los estudiantes. Pero cabe advertir que, habiendo tenido un origen metódico, la división de la Historia se beneficia actualmente con los resultados obtenidos por las ciencias que estudian el origen y desarrollo de las formas sociales. $Y$ aunque no pueda estimárselos como definitivos, algunos recientes ensayos de división de la Historia (Weber, por ejemplo) muestran mayor espíritu de crítica y síntesis que las divisiones tradicionales.

a), según el grado de certeza de los conocimientos históricos, el campo de la Historia suele ser limitado en la siguiente forma: -Prehistoria, cuyas fuentes están constituídas únicamente por los restos materiales;

-Protohistoria, que comprende aquellos hechos legendarios de los cuales se ha recibido oscuras noticias mediante la tradición oral;

--Historia, cuyo náoimiento coincide con la invención de la escritura, a la cual se debe la posibilidad de trasmitir fielmente la relación de los sucesos.

b), en atención al tiempo en que se produjeron los hechos estudiados por ella, la Historia propiamente dicha se divide en cuatro edades, a saber:

- Antigua, desde la formación de los primeros pueblos hasta la división del Imperio Romano (396);

- Media, desde la división del Imperio Romano hasta la toma de Constantinopla por los turcos (1453) o, según otros, hasta el descubrimiento de América;

- Moderna, desde la toma de Constantinopla por los turcos hasta la revolución francesa (1789); y

-Contemporánea, desde la revolución francesa hasta nuestros días. 
c), en conformidad con la mayor o menor extensión del área geográfica en que se desarrollan los hechos históricos, se distinguen:

- Historia Universal, que afecta a todos los pueblos del mundo; - Historia General, limitada a los principales pueblos del mundo, $O$ a un continente; y

-Especial, o monográfica, extendida únicamente a la historia de un país determinado (aunque, a decir verdad, se aplica estas últimas denominaciones a los estudios históricos que atañen a una época determinada, o a un fenómeno).

Aceptada hasta el siglo XIX, la división de la Historia en cuatro edades sucesivas, es hoy objeto de insistentes censuras: 19, porque es artificial suponer que la evolución del mundo experimenta un cambio definitivo y total en una fecha determinada que a la sumqi representa la culminación de un proceso histórico iniciado mucho tiempo antes, o el comienzo de un fenómeno que sólo madurará con el curso de los años; 2 ', porque la denominación de las épocas induce a serios equívocos, en tanto que entre los contemporáneos incluye hechos bastante alejados de nuestra experiencia, como modernos clasifica hechos con caracteres harto superados, etc.; y $3^{\circ}$, porque, en buena cuenta, es una división que atiende al desarrollo histórico de Europa y, para adaptarla al estudio de las otras partes del mundo, es menester forzar, a veces con exceso, los caracteres de la evolución cumplida en éstas.

Al efectuar una división de los hechos pasados, muchos historiadores se rijen actualmente por las formas sociales predominantes o por los avances de la técnica. Aunque de manera elemental, Condorcet siguió esos índices al fijar las grandes etapas del progreso humano. Y a ellos se atiende, universalmente, para estudiar la prehistoria, la sociedad primitiva y el desenvolvimiento económico-social de los pueblos históricos, porque las formas sociales y la técnica determinan, en su totalidad, el complejo cultural $\mathrm{y}$, por lo tanto, proporcionan bases estables para determinar el estadio de la evolución histórica y el ritmo de desarrollo de cada pueblo en particular. Atendiendo a tales índices se distingue: $1^{\circ}$, comunismo primitivo; $2^{\circ}$, esclavitud; $3^{\circ}$, feudalismo; $4{ }^{\circ}$, capitalismo, y 5 , socialismo.

Formas de la Historia.-La tarea del historiador se limitó un tiempo a restaurar la apariencia de los hechos pasados, o a deducir de éstos las enseñanzas morales que convenían a la educación de los gobernantes y los pueblos, y fué usual considerar entonces que la Historia podía 
ser pintoresca o filosófica. A la primera atendían los retores, cuando diferenciaban las diversas formas que la Historia adopta, según la modalidad de la exposición y la extensión de los hechos tratados. Y, aunque tales formas corresponden a una época en que la Historia era considerada como género literario, se las debe conocer: porque las obras históricas del pasado se adecuaron a esas formas y su debido aprovechamiento exige tenerlas en cuenta; y porque algunas de ellas tienen vigencia hasta nuestros días.

Atendiendo a la modalidad de la exposición, se tiene:

a), crónica, que ordena los hechos pasados conforme a lis sucesion en el tiempo (por ejemplo, las cronicas de la conquista del Peru);

b), anales, en los que se relata los hechos ocurridos de año en año (por ejemplo, Anales del Perú por Fernando de Montesinos);

c) diarı, que se carücteriza por referir los hechos de día en día (por ejemplo, Liario ae Lirna por Francisco y Jose de Mugaburu);

d), elemérides, es una especie de calendario histórico, en el cual se enuncian los hecros que, en cada dia del año, han ocurrido a través de todos los tiempos.

$Y$, atendiendo a la extensión de los hechos tratacios, tenernos:

a), tastos, estudio historico limitacio a los sucesos acaecidos durante un gobierno;

b), paralelo, que tieñe por Objetó confrontar lass semejanzas y diferencias de los personajes representativos de dos pueblos (como lo hiciera Plutarco en sus Vidas Paralelas), o comparar el carácter y la conducta histórica de dos personajes representativos de un momento dado (como lo hace Jorge Basadre al comparar a Castilla y Vivanco, en su Historia de la República);

c), biografía, que sigue la trayectoria histórica de un personaje notable, a través de todos los actos de su vida; y

d), memorias, en las cuales el relato histórico es hecho por los personajes que hubiesen actuado en los hechos tratados.

Valor cultural de la Historia.-Ha existido la tendencia de considerar la Historia como ciencia cultural, para establecer, así, una diferencia esencial entre ella y las ciencias susceptibles de llenar una finalidad práctica. Pero, en tanto que su máximo objetivo es el desarrollo de la civilización, la Historia es una ciencia de ciencias; $y$, en verdad, no se concibe 
el actual estadio de la evolución social y cultural sin la contribución de la Historia, que ha conservado la noticia de todos los progresos humanos $y$ ha permitido superarlos.

El conocimiento de la Historia no queda constreñido a proporcionar los recursos gratos a la erudición y la elocuencia. En sus fases especiales (que pueden referirse a una ciencia, una institución, etc.) es síntesis de progreso y perspectiva para el futuro. $Y$ en sus fases generales (que reconstruyen la dinámica del desarrollo de los pueblos), es elemento que ayuda a los hombres y a los pueblos en la consciente determinación de su conducta histórica.

ALBERTO TAURO.

\section{Bibliografía}

Correspondiente a la parte preliminar de INTRODUCCION A LA HISTORIA UNIVERSAL.

Al utilizarla, el alumno debe tener presente:

19, que no incluye las obras de los autores mencionados en el Programa razonado; $y$

2. que está obligado a conocer por lo menos dos de los libros incluídos en esta Bibliogratia, y que el examen pueda basarse en la exposición y crítica de sus respectivos contenidos.

BARNES, Harry Elmer. A History of the historical writing. Norman (Oklahoma), University of Oklahoma Press, 1938.

BAUER, Guillermo.-introducción al estudio de la Historia. Trad. de la $2^{\alpha}$ ed. alemana y notas por Luis G. de Valdeavellano. Barcelona, Casa Editoral Bosch, 1944.

BUJARIN, Nicolás.-El materialismo histórico. Madrid, Editorial Cenit S. A., 1933.

BURCKHARDT, Jacobo.-Retlexiones sobre la Historia Universal. Prólogo de Alfonso Reyes. México, Fondo de Cultura Económica, 1943.

CASO, Antonio.-El concepto de la Historia Universal y la Filosofía de los Valores. México, Ediciones Botas, 1933.

CASSIRER, Ernst.-Antropología Filosófica. Introducción a una Filosofía de la Cultura. México, Fondo de Cultura Económica, 1945.

CROCE, Benedetto.-La Historia como hazaña de la libertad. México, Fondo de Cultura Económica, 1942.

FEDERN, Karl.-La concepción materialista de la Historia. Traducida por Carlos María Reyles. Buenos Aires, Espasa Calpe Argentina, 1942. 
FERRATER MORA, José.-Cuatro visiones de la Historia Universal. Buenos Aires, Editorial Losada S. A., 1945.

GOOCH, G. P.-Historia e historiadores en el siglo XIX. Versión española de Ernestina de Champourcin y Ramón Iglesia. México, Fondo de Cultura Económica, 1942.

HUIZINGA, J.-Sobre el estado actual de la ciencia histórica. Madrid, Revista de Occidente, 1934.

LANGLOIS, C. V. (y Charles Seignobos).--Introducción a los estudios históricos. Trad. por Domingo Vaca. Madrid, Daniel Jorro, 1913.

LE BON, Gustavo.--Bases científicas de una Filosofía de la Historia. Trad. de F. García. Madrid, M. Aguilar, 1931.

MEINECKE, Friedrich.-El historicismo y su génesis. México, Fondo de Cultura Económica, 1943.

SCHNEIDER, Hermann.-Filosofía de la Historia. Trad. por José Rovira Armengol. Barcelona-Buenos Aires, Editorial Labor S. A., 1931.

SEIGNOBOS, Charles.-El método histórico aplicado a las ciencias sociales. Trad. por Domingo Vaca. Madrid, Daniel Jorro, 1923.

SELIGMAN, Edwin R. A.-La interpretación económica de la Historia. Estudio preliminar de Adolfo Posada. Madrid, Francisco Beltrán, 1929.

SHOTWELL, J. T.-Historia de la Historia en el mundo antiguo. México, Fondo de Cultura Económica, 1940.

VIERKANDT, Alfredo.-Filosofía de la sociedad y de la Historia. Prólogo de Ricardo-Levene. La Plata, Biblioteca de la Universidad de La Plata, 1934.orge Puccinelli Converso»

XENOPOL, A. D.-Teoría de la Historia. 2a ed. Trad. de Domingo Vaca. Madrid, Daniel Jorro, 1911. 